
\title{
3 Research Square \\ Ferroptosis inhibitor alleviates cytotoxicity induced by cobalt nanoparticles through reactive oxygen species attenuation
}

\section{Chen Wang (D 574988122@qq.com)}

Nantong University Affiliated Hospital: Affiliated Hospital of Nantong University https://orcid.org/00000002-6063-3200

\section{Weinan Zhang}

Nantong University Affiliated Hospital: Affiliated Hospital of Nantong University

\section{Fan Liu}

Nantong University Affiliated Hospital: Affiliated Hospital of Nantong University

\section{Yake Liu}

Nantong University Affiliated Hospital: Affiliated Hospital of Nantong University https://orcid.org/00000002-2665-8914

\section{Research Article}

Keywords: total hip arthroplasty, cobalt nanoparticles, ferroptosis, ferroptosis inhibitor, ROS

Posted Date: June 24th, 2021

DOI: https://doi.org/10.21203/rs.3.rs-619496/v1

License: (c) (1) This work is licensed under a Creative Commons Attribution 4.0 International License. Read Full License 


\section{Abstract}

Background Currently, tissue damage induced by cobalt nanoparticles (CoNPs) are the most noticeable adverse effect in the patients with metal-on-metal (MOM) hip prostheses. Therefore, effective therapeutic strategy is needed to limit the toxicity of CoNPs. In this study, we aimed to investigate the protective effect of ferroptosis inhibitor on CoNPs-induced cytotoxicity.

Methods To evaluate the detoxification effect of ferroptosis inhibitor, MG-63 cells were treated with CoNPs and ferroptosis inhibitor for $24 \mathrm{~h}$. The cell viabilities were measured by CCK- 8 assay. The expressions of glutathione peroxidase 4(GPX4) and solute carrier family 7 member 11(SLC7A11) were examined by western blot. Reactive oxygen species (ROS) and the GSH level were measured by the relevant kits. The cell apoptosis was detected by flow cytometry. The serum levels of inflammatory cytokines were measured with Elisa.

Results The cytotoxic effects of CoNPs were time- and dose-dependent. In addition, CoNPs elicited a significant $(p<0.05)$ reduction in cell viability and the glutathione $(\mathrm{GSH})$ level with a concomitant increase in ROS generation and inflammatory cytokines such as tumour necrosis factor a (TNF- $\alpha)$, interleukin-1 $\beta$ (IL-1 $\beta$ ) and interleukin-6 (IL-6) after 24 hours of exposure. Deferiprone (DFP) provided significant protection for cells after treatment with CoNPs. Western blot analysis demonstrated that CoNPs decreased the expression of GPX4 and SLC7A11, and treatment with DFP inhibited the decrease.

Conclusions CoNPs were highly cytotoxic and ferroptosis inhibitor DFP has a detoxification effect on cytotoxicity induced by CoNPs. Ferroptosis plays an important role in the process that CoNPs induced the cytotoxicity. The effectiveness of ferroptosis inhibition on MG-63 cells may initiate the development of novel strategies for reducing the adverse effects associated with metal implants in future.

Trial registration: no human participant.

\section{Background}

Total knee arthroplasty (TKA) and total hip arthroplasty (THA) are among the most effective orthopedic surgeries that can significantly restore joint function, relieve pain, and improve the patient's standard of living [1]. After total hip replacement, many different reasons lead to the production of metal particles, mainly due to mechanical wear and biological environmental erosion, such as erosion of the prosthesis by fluid and tissues around the hip joint. Cobalt-containing metal alloys release a large number of metal debris particles and metal ions[2]. Due to various of metal particles generated by wear, CoNPs may spread throughout the body, causing toxic effects around the implants. Horev-Azaria et al. believe that the toxicity of CoNPs is caused by the dissolution of $\mathrm{Co}^{2+}$ in the nanoparticles. However, previous researches have confirmed that the toxicity of nanoparticles themselves is greater than that of ionic forms $[3,4]$. Many studies have shown that exposure to CoNPs can cause oxidative stress, lung inflammation and cell proliferation, which in turn leads to DNA damage and DNA mutations [5]. In vitro studies have indicated that CoNPs result in cellular oxidative stress [6] and genotoxicity [7]. Nanoparticles is known to enter 
human cells and induce the production of reactive oxygen species, thereby leading to cytotoxicity and genotoxicity [8]. In the cytoplasm, CoNPs can affect the mitochondrial redox reaction, generate a large number of ROS, and consume intracellular antioxidants such as GSH, in turn, induce redox stress and cause damage to the cell structure [9]. Excessive ROS production leads to the damage of mitochondria, lysosomes and nuclei, and ultimately activates the apoptosis pathway [10]. In addition, pro-inflammatory cytokines such as TNF- $a$, IL-1 $\beta$, and IL- 6 were found in the tissues surrounding the necrotic implants [11, 12]. Therefore, it is important to determine how to protect tissues from oxidative stress and inflammation.

The term ferroptosis was proposed by Dixon et al. in 2012 to describe the form of cell death induced by small molecules such as erastin [13]. Ferroptosis is dependent upon intracellular iron, accumulation of lipid ROS, and loss of activity of the lipid repair enzyme GPX4. Ferroptosis is a recently recognized form of regulated cell death, involving iron accumulation and lipid peroxidation, and its death phenotype is different from other types of cell death (such as apoptosis and necrosis) [14]. The depletion of GPX4, the down-regulation of SLC7A11 activity and the increase of lipid peroxidation products are common features of ferroptosis $[15,16]$. At the same time, GPX4 is also an important regulator of ferroptosis [17]. The expression of SLC7A11 can potentially improve the survival rate of cells by regulating iron metabolism[18]. In addition, SLC7A11 is not only an essential component of the amino acid antiporter system $\mathrm{Xc}^{-}$, but also plays a crucial protective role in GSH synthesis. Since its recognition, ferroptosis has been involved in degenerative diseases such as the kidney and brain, and plays an important role in a variety of cancers [19]. The accumulation of reactive oxygen species plays a key role in causing ferroptosis [20], which is also the main trigger for the toxicity of CoNPs. In addition, ferroptosis inhibitors have been shown to have anti-inflammatory effects by inhibiting the expression of inflammatory cytokines including TNF-a[21], and the inflammatory response is also one of the toxic factors of CoNPs. Therefore, we speculate that ferroptosis inhibitors can reduce the toxicity of CoNPs based on inhibition of inflammation and ROS.

As far as we know, there is no previous study on ferroptosis inhibitors to antagonize the toxicity of cobalt nanoparticles. In this study, we explored the iron chelator DFP as an ferroptosis inhibitor to antagonize the toxicity of cobalt nanoparticles by depletion of ROS. This experiment aims to explore the possible protective effects of ferroptosis inhibitors on the toxicity of cobalt nanoparticles. Finally, we suggest that DFP, as an ferroptosis inhibitor, can be used as a therapeutic agent to prevent toxicity after MOM artificial joint replacement.

\section{Materials And Methods}

\section{Reagent and chemicals}

CoNPs (30nm,99.9\%) were produced by Shanghai Chaowei Nano Technology Company. Dulbecco's Modified Eagle's medium (DMEM), 2', 7'-dichlorofluorescin diacetate (DCFH-DA), fetal bovine serum (FBS), trypsin-eDtA and penicillin/streptomycin were purchased from Gibco (Life Technologies, Paisley, UK). Tissue culture dishes were obtained from corning Inc. (New York, New York). Cell counting kit-8 (CCK- 
8), ROS detection kit, were purchased from Beyotime Company (Jiangsu, China). Mouse TNF-a, IL-1 $\beta$, and IL-6 enzyme-linked immunosorbent assay (ELISA) kits were purchased from R\&D Systems (Minneapolis, MN). Annexin V-FITC was purchased from Multi Sciences (Hanzhou, China). All antibodies were purchased from Abcam (Cambridge, UK).10\% polyacrylamide gel premix (NCM FastPAGE) was purchased from New Cell\&Molecular Biotech Co.,Ltd (Suzhou, China). Deferiprone was gotten from MCE (New Jersey, USA).

\section{Preparation of Cobalt nanoparticles}

CoNPs samples were sterilized at $180^{\circ} \mathrm{C}$ for $4 \mathrm{~h}$, suspended in ultrapure water to achieve the initial concentration of $40 \mathrm{mM}$. The Freshly prepared stock solution was ultrasonicated at room temperature at $40 \mathrm{~W}$ for $30 \mathrm{~min}$ in order to minimize particle aggregates and diluted to achieve the required concentrations $(25 \mu \mathrm{M}, 50 \mu \mathrm{M}, 100 \mu \mathrm{M}, 200 \mu \mathrm{M}, 400 \mu \mathrm{M}, 800 \mu \mathrm{M})$ with complete culture medium.

\section{Preparation of DFP}

DFP $(1 \mathrm{mM})$ was irradiated and sterilized under ultraviolet light for $2 \mathrm{~h}$, and then diluted to $25 \mu \mathrm{M}$ with basal medium for subsequent experiments. Store the processed DFP at $-20^{\circ} \mathrm{C}$.

\section{Cell culture and sample treatment}

The MG-63 cell line was bought from the cell repository of Chinese Academy of Sciences. Briefly, cells were cultured in DMEM supplemented with 10\% FBS, penicillin and streptomycin. The medium was replaced every 3 days. All cells were grown at $37^{\circ} \mathrm{C}$ in a humidified incubator containing $5 \% \mathrm{CO}_{2}$. For the dose-related experiments, the cells were exposed to CoNPs for 4, 24 and $48 \mathrm{~h}$. To examine the protective effects of DFP on the viability of cells, the cells were treated with varying concentrations of DFP and exposed to target concentration of CoNPs for $24 \mathrm{~h}$.

\section{Cell Viability Assay}

According to the manufacturer's protocol, MG-63 cells were incubated in 96-well plates and then treated with different concentrations of DFP and $400 \mu \mathrm{M}$ CoNPs for 4, 24 or $48 \mathrm{~h}$. After that, $10 \mu \mathrm{L}$ CCK-8 was added to each well and incubated at $37{ }^{\circ} \mathrm{C}$ for another $4 \mathrm{~h}$. The absorbance was measured by the microplate reader at $450 \mathrm{~nm}$. The Cell viability ratio was expressed as percent control. From this, the $50 \%$ inhibitory concentration (IC50 value)[22] was calculated.

\section{Detection of Cytokines}

Cells were plated at a density of $1 \times 10^{5}$ cells/well in six-well plates and incubated for $24 \mathrm{~h}$ at $37^{\circ} \mathrm{C}$. Then the cells were exposed to CoNPs $(400 \mu \mathrm{M})$ with or without the treatment of DFP $(25 \mu \mathrm{M})$ for $24 \mathrm{~h}$. After treatment, cytokines levels of the supernatants collected from cell cultures were measured. The concentrations of TNF-a $\square \mathrm{IL}-1 \beta \square I \mathrm{~L}-6$ in the culture media were determined using ELISA kits according to the manufacturer's instructions. Each sample was analyzed in triplicate.

\section{ROS measurement}

Spectrofluorometry analysis and fluorescence microscopy imaging were used to assess ROS generation in cells exposed to target concentrations of CoNPs and the protective effects of DFP treatment. For the 
spectrofluorometry analysis, cells $\left(1 \times 10^{5} /\right.$ well) were seeded in 96 -well plates. MG-63 cells were exposed to CoNPs and ferroptosis inhibitor for $24 \mathrm{~h}$. Following completion of the DCFH-DA reaction, the reaction mixture was washed thrice by $200 \mathrm{ml}$ of PBS in each well. The fluorescence intensity of the suspension was measured on a multi-well microplate reader using excitation and emission of $485 \mathrm{~nm}$ and $525 \mathrm{~nm}$, respectively. Values were expressed as the percentage of fluorescence intensity relative to the control wells.

\section{GSH Measurement}

The cells were treated with $25 \mu \mathrm{M}$ DFP and exposed to $400 \mu \mathrm{M}$ CoNPs for $24 \mathrm{~h}$. After the exposure, the cells were washed with PBS twice, scraped off, suspended in PBS, and centrifuged at $1000 \times \mathrm{g}$. The cell pellet was then homogenized in 5\% 5-sulfosalicylic acid. Next, the suspension was lysed by freezing and thawing twice after 5 min centrifuged at 10,000×g for $10 \mathrm{~min}$. The amount of GSH were measured according to the manufacturer's protocol.

\section{Quantitative assessment of cell apoptosis detected by flow cytometry}

For measurement of cell apoptosis, the AnnexinV-FITC assay kit was conducted according to manufacturer's protocol. Briefly, cells were washed with PBS solution and harvested. Next, the cells were suspended in binding buffer at $1 \times 10^{6}$ cells $/ \mathrm{ml}$. A $100 \mathrm{ml}$ cell suspension was withdrawn supplemented with $5 \mathrm{ml}$ AnnexinV-FITC and $10 \mathrm{ml}$ of PI and subsequently incubated for $15 \mathrm{~min}$ at room temperature in the dark. A Becton Dickinson flow cytometer was employed for the measurement within $1 \mathrm{~h}$ of staining.

\section{Western Blot}

The cells were treated with $25 \mu \mathrm{M}$ DFP and exposed to target concentrations of CoNPs for $24 \mathrm{~h}$. Cells were washed in cold PBS and lysed in RIPA with $1 \mathrm{mM}$ phenylsulfonyl fluoride (PMSF, beyotime, Nantong, China). The supernatants were harvested and protein concentrations were measured with the bicinchoninic acid (BCA) assay kit. The concentrated samples were subjected to $15 \%$ SDS-

polyacrylamide gel electrophoresis and transferred to a poly-vinylidene diflouride filter (PVDF) membrane by a transfer apparatus at $300 \mathrm{~mA}$ for $1 \mathrm{~h}$. The membranes were blocked for $1 \mathrm{~h}$ and then incubated with primary antibodies at $4^{\circ} \mathrm{C}$ overnight. After washing with TBST, the membranes were incubated with secondary antibodies for $2 \mathrm{~h}$ at room temperature. The PVDF membranes were washed four times with TBST according to the manufacturer's protocol.

\section{Statistical Analysis}

All statistical analyses were performed using GraphPad Prism software 7 (GraphPad Software Inc., La Jolla, CA, USA) using one-way analysis of variance (ANOVA) followed by Dunnett's test to evaluate significance relative to control. Results shown summarise data from three independent experiments and were represented as the mean \pm standard deviation (SD). Differences between groups were considered significant if $p$-values $<0.05$.

\section{Results}




\section{Ferroptosis inhibitor protected against cytotoxicity induced by CoNPs in MG-63 cells}

Cells were treated with different concentrations of CoNPs for $4 \mathrm{~h}, 24 \mathrm{~h}$ or $48 \mathrm{~h}$ and the effect of CoNPs on MG-63 cell survival was determined by CCK-8 assay. The percentage of survived cells strikingly decreased with the increase of CoNPs concentration (Fig. 1a). The IC50 value was $400 \mu \mathrm{M} 24 \mathrm{~h}$ later, respectively. Cells cultured in the medium with varying concentrations of DFP, which showed a dose-dependent survival rate. When the concentration of DFP increased, the viability of MG-63 cells increased. When cells were cultured in $400 \mu \mathrm{M}$ CoNPs and different concentrations of DFP, the viability of cells was significantly higher compared with the cells treated with CoNPs (Fig. 1b).

\section{Ferroptosis inhibitor reduced inflammatory cytokines in MG-63 cells}

The results are shown in the Fig. 2. The levels of TNF-a, IL-1 $\beta$ and IL-6 in the CoNPs group were noticeably higher than those in the control group. Compared with the CoNPs group, DFP treatment had a significant protective effect, and significantly reduced TNF- $a(p<0.05), I L-1 \beta(p<0.05)$ and IL-6 $(p<0.05)$ induced by cobalt nanoparticles expression.

\section{ROS formation in MG-63 cells}

The oxidative damage induced by ROS is considered to be an important cause of the toxicity of cobalt nanoparticles. ROS is also the main incentive of ferroptosis. In this study, as shown in the Fig. 3, after 400 $\mu \mathrm{M}$ CoNPs were treated for $24 \mathrm{~h}$, the ROS level of MG-63 cells increased significantly $(p<0.05)$, while DFP $(25 \mu \mathrm{M})$ could significantly inhibit this increase $(p<0.05)$, and the difference was statistically significant $(p<0.05)$.

GSH is the key cellular endogenous antioxidant that can scavenge toxic free radicals, and depletion of GSH appears to promote intracellular ROS accumulation, leading to apoptosis. Altered GSH levels represent increased cellular response to oxidative stress. The results suggested that CoNPs treatment decreased GSH levels $(p<0.05)$ and DFP inhibited this decrease $(p<0.05)$ as measured by the GSH assay kit (Fig. 3c).

\section{Apoptosis assay in MG-63 cells}

To investigate the apoptosis rate of cells treated with $400 \mu \mathrm{M}$ CoNPs and $25 \mu \mathrm{M}$ DFP, the AnnexinV-FITC assay kit was used. Incubation with $400 \mu \mathrm{M}$ CoNPs for $24 \mathrm{~h}$ mildly increased the cell apoptosis rate compared with the control group. When DFP was added as a treatment before exposure to CoNPs, the apoptosis was slightly alleviated (Fig. 4a, b).

\section{Ferroptosis inhibitor exerted the positive regulation on GPX4 in MG-63 cells}

GPX4 is the central regulator of ferroptosis, and the decline of GPX4 is often used as a marker of ferroptosis[23-25]. SLC7A11 is a unit of the glutamate-cystine antiporter $\mathrm{Xc}^{-}$, resulting in enhanced lipid oxidation and ferroptosis. Western blot showed that CoNPs noticeably decreased the protein amount of GPX4 and SLC7A11. However, the decrease in GPX4 and SLC7A11 levels was inhibited when cells were treated with $25 \mu \mathrm{M}$ DFP and $400 \mu \mathrm{M}$ CoNPs for $24 \mathrm{~h}$. These results suggest that CoNPs might induce ferroptosis and the ferroptosis inhibitor DFP exerts therapeutic action against CoNPs. 


\section{Discussion}

With the gradual popularity of artificial joint replacement, the materials of artificial joint prostheses have also attracted much attention. Due to the low dislocation and wear rate of MOM implants, their use in prostheses has gradually become widespread. It is crucial to elucidate the adverse biological consequences of metal nanoparticles released from implants, and provide a potential method for reducing the adverse effects associated with these metal particles. The main adverse effects come from cobalt nanoparticles produced under long-term wear and tear. So far, the mechanism of CoNPs-induced toxicity has not been fully understood, and the current mainstream is still the ROS theory and the inflammation caused by ROS[26, 27]. The excessive accumulation of ROS and the production of a large number of inflammatory cytokines such as TNF-a play an important role in the toxicity mechanism induced by cobalt nanoparticles, leading to inflammation, cell damage and proliferation, which in turn leads to DNA damage and DNA mutations, as well as damage to mitochondria, lysosomes and the nucleus, eventually leading to cell death. Past studies have found that although the apoptosis rate of treatment is significantly higher than that of the control group, it is still much lower than the cell death rate[28]. Therefore, it could be possible that there are still other types of cell death besides apoptosis in MG-63 cells exposed to CoNPs. Ferroptosis is a newly identified form of cell death that is characterized by the accumulation of intracellular iron and the need for lipid peroxidation[14]. In this process, a variety of inducers disrupt the cell redox balance and produce a large amount of lipid peroxidation products, which ultimately trigger cell death. Generally speaking, ferroptosis is a form of ROS-dependent regulatory necrosis that occurs through multiple signals and pathways[29]. In this study, we focused on the role of ferroptosis in toxicity induced by cobalt nanoparticles and evaluate the protective effects of ferroptosis inhibitors on MG-63 cells.

In current study, the CCK8 was used to assess the viability of MG-63 cells exposed to CoNPs. The results showed that the cytotoxic effect of CoNPs on MG-63 cells was time- and dose-dependent. On this basis,

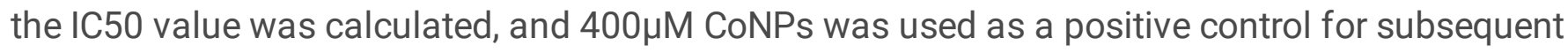
experiments. In addition, $25 \mu \mathrm{M}$ DFP was selected to evaluate its effect on CoNPs-induced cell damage. Our results suggest that DFP treatment can reduce the decline in cell viability induced by CoNPs. The cell viability of the experimental group treated with the ferroptosis inhibitor was much higher than that of the control group, which indicated that the ferroptosis inhibitor had a certain protective effect on MG-63 cells.

Studies have shown that cobalt-chromium wear particles can induce the release of a variety of proinflammatory cytokines, such as TNF-a, IL-1 $\beta$, and IL-6[30-32]. In this study, we confirmed the proinflammatory effect of CoNPs through the increase of TNF- $\alpha$, IL-1 $\beta$ and IL-6, and determined whether ferroptosis inhibitors can reduce the increase in the levels of inflammatory cytokines induced by CoNPs. The treatment of ferroptosis inhibitor significantly reduced the inflammatory cytokines induced by CoNPs after 24 hours of exposure, and the difference was statistically significant compared with the control group $(P<0.05)$. DFP treatment can significantly reduce the expression of TNF- $a$, IL-1 $\beta$ and IL- 6 in MG-63 cells induced by $400 \mu \mathrm{M}$ CoNPs, suggesting that ferroptosis inhibitors have a protective effect on inflammatory cytokines in MG-63 cells induced by $400 \mu \mathrm{M}$ CoNPs. 
CoNPs induced a noticeable increase in ROS. ROS is considered to be the main cause of ferroptosis, which can cause DNA damage, oxidative stress and activate other cellular processes. On the one hand, ROS acts as a dynamic balance signal molecule that regulates cell growth, and on the other hand, it acts as an adaptive response at physiological concentrations. However, the production of ROS at higher concentrations can cause cell damage and death. GSH is an important antioxidant substance that plays an important role in protecting against apoptosis. The depletion of GSH promotes the accumulation of ROS in cells and cell apoptosis. In current study, compared with CoNPs group, the addition of ferroptosis inhibitors significantly reduced the production of ROS in MG-63 cells(Fig. 3a, b). In addition, our results shows that CoNPs treatment decreased GSH levels and DFP reversed this decrease $(p<0.05)$ as measured by the GSH assay kit (Figure.3c).

Flow cytometry results exhibited that CoNPs induced mild cellular apoptosis, which could be further alleviated by the exposure of DFP. Although the apoptosis rate of MG-63 cells for the CoNPs treatment group was significantly increased as compared to the control group (Fig. 4), it was still much lower than that of control group, as shown by CCK-8 under the same conditions (Fig. 1). These results indicate that non-apoptotic mechanisms are involved in CoNPs-mediated cell death.

GPX4 and SLC7A11 are recognized as important endogenous regulators of ferroptosis and the decline of GPX4 and SLC7A11 is always regarded as a marker of ferroptosis[33, 34]. A previous study has indicated that SLC7A11 protects cells from ferroptosis by increasing GSH production. In this study, we found that after exposure to CoNPs, the protein levels of GPX4 and SLC7A11 were significantly decreased, while DFP up-regulated the levels of GPX4 and SLC7A11, reducing the damage of ROS. Our results strongly support the idea that ferroptosis inhibitors can upregulate the expression of GPX4 and SLC7A11 to inhibit the cytotoxicity caused by CoNPs.

\section{Conclusions}

In summary, all these results strongly supported the idea that the ferroptosis inhibitor counteracts cytotoxicity and inflammation induced by CoNPs and ferroptosis plays an important role in the occurrence of CoNPs-induced toxicity. In addition, we are supposed to compare the results of different ferroptosis inhibitors on cell detoxification, and further screen out the most suitable drugs to relieve the CoNPs-induced toxicity produced in MOM artificial joints. The research provides a new perspective for antagonizing CoNPs-induced cytotoxicity, and provides a potential method to reduce adverse reactions related to MOM joint implants.

\section{Abbreviations}

CoNPs: cobalt nanoparticles; MOM: metal-on-metal; GPX4: glutathione peroxidase 4; SLC7A11: solute carrier family 7 member 11; ROS: Reactive oxygen species; GSH: glutathione; TNF-a: tumour necrosis factor $\alpha$; IL-1 $\beta$ : interleukin-1 $\beta$; IL-6: interleukin-6; DFP: Deferiprone; TKA: Total knee arthroplasty; THA: total hip arthroplasty; DCFH-DA: 2', 7'-dichlorofluorescin diacetate; DMEM: Dulbecco's Modified Eagle's 
medium;FBS: fetal bovine serum; CCK-8: Cell counting kit-8; PMSF: phenylsulfonyl fluoride; PVDF: polyvinylidene diflouride filter;SD: standard deviation

\section{Declarations}

Competing interests: The authors declare that they have no conflicting interests.

Funding: This work was supported by Postgraduate Research \& Practice Innovation Program of Jiangsu Province [grant number SJCX20_1167].

Author details: ${ }^{1}$ Department of Orthopaedics, Affiliated Hospital of Nantong University, Nantong, Jiangsu Province, China 226001

Authors' contributions: CW and WZ researched literature and conceived the study. YL was involved in protocol development and data analysis. CW wrote the first draft of the manuscript. All authors reviewed and edited the manuscript and approved the final version of the manuscript

Acknowledgements: Not applicable

Availability of data and materials: The datasets used and/or analyzed during the current study are not publicly available. Data are however available from the corresponding author on reasonable request.

\section{References}

1. $\mathrm{O}, \mathrm{E}$. , et al., Health-related quality of life in total hip and total knee arthroplasty. A qualitative and systematic review of the literature. 2004. 86(5): p. 963-74.

2. Jacobs, J., et al., Metal degradation products: a cause for concern in metal-metal bearings? 2003(417): p. 139-47.

3. Sabbioni, E., et al., Cytotoxicity and morphological transforming potential of cobalt nanoparticles, microparticles and ions in Balb/3T3 mouse fibroblasts: an in vitro model. Nanotoxicology, 2014. 8(4): p. 455-464.

4. Papageorgiou, I., et al., The effect of nano- and micron-sized particles of cobalt-chromium alloy on human fibroblasts in vitro. Biomaterials, 2007. 28(19): p. 2946-2958.

5. Wan, R., et al., Cobalt nanoparticles induce lung injury, DNA damage and mutations in mice. Part Fibre Toxicol, 2017. 14(1): p. 38.

6. C, M., et al., The pro-inflammatory effects of low-toxicity low-solubility particles, nanoparticles and fine particles, on epithelial cells in vitro: the role of surface area. 2007. 64(9): p. 609-15. 
7. R, C., et al., Comparative genotoxicity of cobalt nanoparticles and ions on human peripheral leukocytes in vitro. 2008. 23(5): p. 377-82.

8. S, R., et al., Comparative cytotoxicity and genotoxicity of cobalt (II, III) oxide, iron (III) oxide, silicon dioxide, and aluminum oxide nanoparticles on human lymphocytes in vitro. 2016. 35(2): p. 17083.

9. Horev-Azaria, L., et al., Predictive toxicology of cobalt nanoparticles and ions: comparative in vitro study of different cellular models using methods of knowledge discovery from data. Toxicological sciences : an official journal of the Society of Toxicology, 2011. 122(2): p. 489-501.

10. M, O., et al., Mitochondria, oxidative stress and cell death. 2007. 12(5): p. 913-22.

11. Masui, T., et al., Expression of inflammatory cytokines, RANKL and OPG induced by titanium, cobalt-chromium and polyethylene particles. Biomaterials, 2005. 26(14): p. 1695-1702.

12. Goodman, S.B., et al., Cellular profile and cytokine production at prosthetic interfaces. Study of tissues retrieved from revised hip and knee replacements. The Journal of bone and joint surgery. British volume, 1998. 80(3): p. 531-539.

13. Dixon, S.J., et al., Ferroptosis: an iron-dependent form of nonapoptotic cell death. Cell, 2012. 149(5): p. 1060-72.

14. Dixon, S.J., et al., Ferroptosis: an iron-dependent form of nonapoptotic cell death. Cell, 2012. 149(5): p. 1060-1072.

15. Han, C., et al., Ferroptosis and Its Potential Role in Human Diseases. Front Pharmacol, 2020. 11: p. 239.

16. Xie, Y., et al., Ferroptosis: process and function. 2016. 23(3): p. 369-79.

17. Yang, W.S., et al., Regulation of ferroptotic cancer cell death by GPX4. Cell, 2014. 156(1-2): p. 317-331.

18. Jiang, L., et al., Ferroptosis as a p53-mediated activity during tumour suppression. 2015. 520(7545): p. 57-62.

19. Zou, Y. and S.L. Schreiber, Progress in Understanding Ferroptosis and Challenges in Its Targeting for Therapeutic Benefit. Cell Chem Biol, 2020. 27(4): p. 463-471.

20. Imai, H., et al., Lipid Peroxidation-Dependent Cell Death Regulated by GPX4 and Ferroptosis. Current topics in microbiology and immunology, 2017. 403: p. 143-170.

21. Zhang, Y., et al., Ferroptosis inhibitor SRS 16-86 attenuates ferroptosis and promotes functional recovery in contusion spinal cord injury. Brain research, 2019. 1706: p. 48-57. 
22. Jiang, H., et al., Effects of cobalt nanoparticles on human $T$ cells in vitro. Biol Trace Elem Res, 2012. 146(1): p. 23-9.

23. Dixon, S., et al., Ferroptosis: an iron-dependent form of nonapoptotic cell death. 2012. 149(5): p. 1060-72.

24. Yang, W., et al., Regulation of ferroptotic cancer cell death by GPX4. 2014. 156: p. 317-331.

25. Friedmann Angeli, J., et al., Inactivation of the ferroptosis regulator Gpx4 triggers acute renal failure in mice. 2014. 16(12): p. 1180-91.

26. Sabbioni, E., et al., Cytotoxicity and morphological transforming potential of cobalt nanoparticles, microparticles and ions in Balb/3T3 mouse fibroblasts: an in vitro model. 2014. 8(4): p. 455-64.

27. Yan, X., et al., alpha-Tocopherol protected against cobalt nanoparticles and coc/2 induced cytotoxicity and inflammation in Balb/3T3 cells. Immunopharmacol Immunotoxicol, 2018. 40(2): p. 179185.

28. Liu, Y., et al., Alpha lipoic acid antagonizes cytotoxicity of cobalt nanoparticles by inhibiting ferroptosis-like cell death. J Nanobiotechnology, 2020. 18(1): p. 141.

29. Lu, L.-Q., et al., Targeting the pathways of regulated necrosis: a potential strategy for alleviation of cardio-cerebrovascular injury. Cellular and molecular life sciences : CMLS, 2021. 78(1): p. 63-78.

30. Thomas, V., et al., In vitro studies on the effect of particle size on macrophage responses to nanodiamond wear debris. 2012. 8(5): p. 1939-47.

31. Hallab, N.J.S., Biologic Responses to Orthopedic Implants: Innate and Adaptive Immune Responses to Implant Debris. 2016: p. S30-1.

32. Kaufman, A., et al., Human macrophage response to UHMWPE, TiAIV, CoCr, and alumina particles: analysis of multiple cytokines using protein arrays. 2008. 84(2): p. 464-74.

33. Imai, H., et al., Lipid Peroxidation-Dependent Cell Death Regulated by GPX4 and Ferroptosis. 2017. 403: p. 143-170.

34. Chen, D., et al., ATF4 promotes angiogenesis and neuronal cell death and confers ferroptosis in a xCT-dependent manner. 2017. 36(40): p. 5593-5608.

\section{Figures}



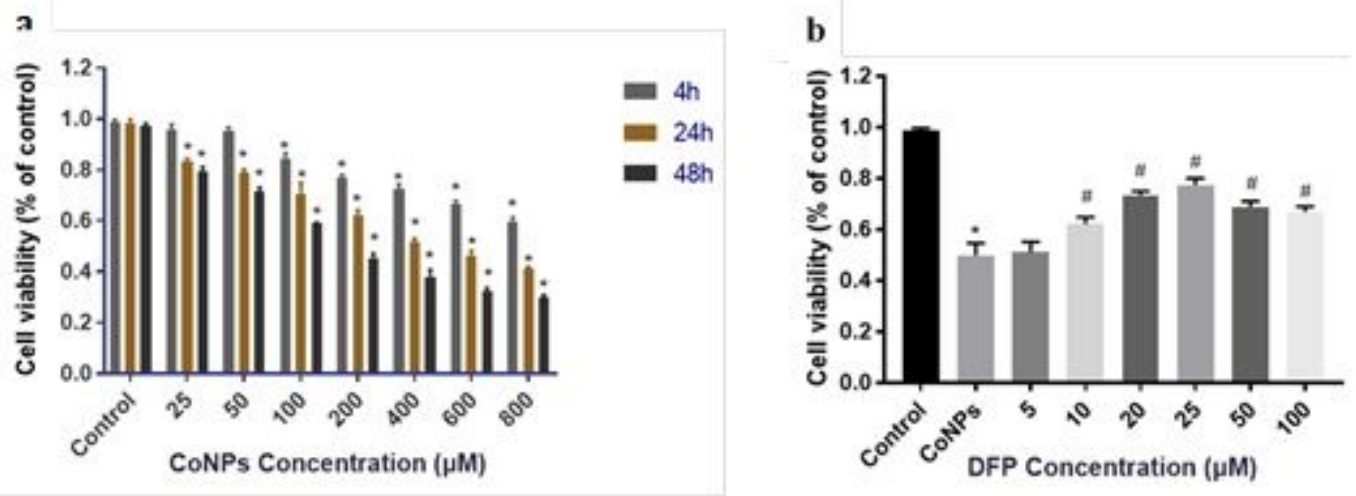

\section{Figure 1}

Changes in cell viability of MG-63 cells following CoNPs and DFP treatment. Cell viability of MG-63 cells exposed to $400 \mu \mathrm{M}$ CoNPs and different concentrations of DFP for $24 \mathrm{~h}$, as determined by a Cell Counting kit 8 assay. Data are presented as the mean \pm standard deviation of three independent experiments performed in triplicate. ${ }^{\star} p<0.05$ vs. control, $\# p<0.05$ vs. CoNPs group.
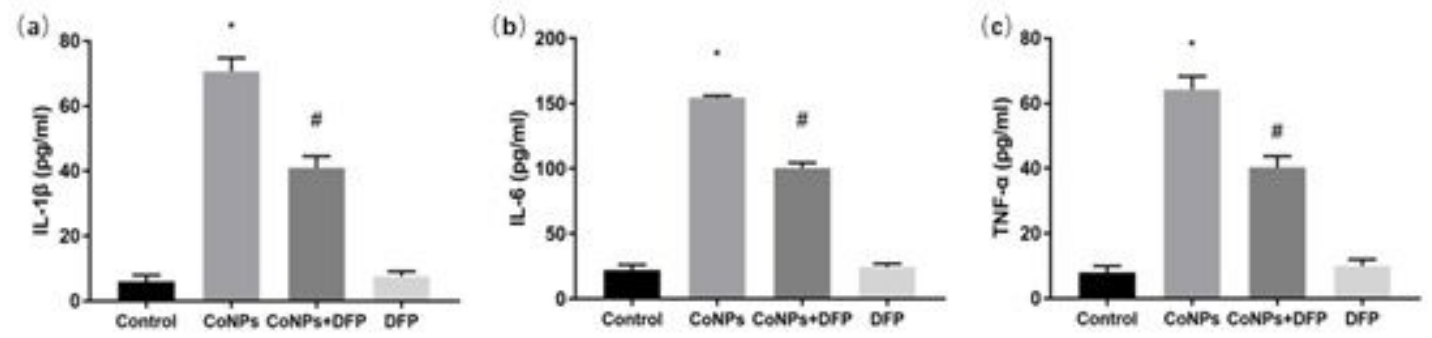

Figure 2

Effects of DFP on pro-inflammatory cytokines and anti-inflammatory cytokines expression in cells in response to exposure to CoNPs. IL-1 $\beta$ (a), IL-6 (b), TNF-a (c) levels in the supernatants of tissue cultured cells after $24 \mathrm{~h}$ of incubation, as evaluated by ELISA. The results are shown as the mean \pm SD of three independent experiments performed in triplicate. ${ }^{*} p<0.05$ compared to control group; $\# p<0.05$ compared to CoNPs group. 
a
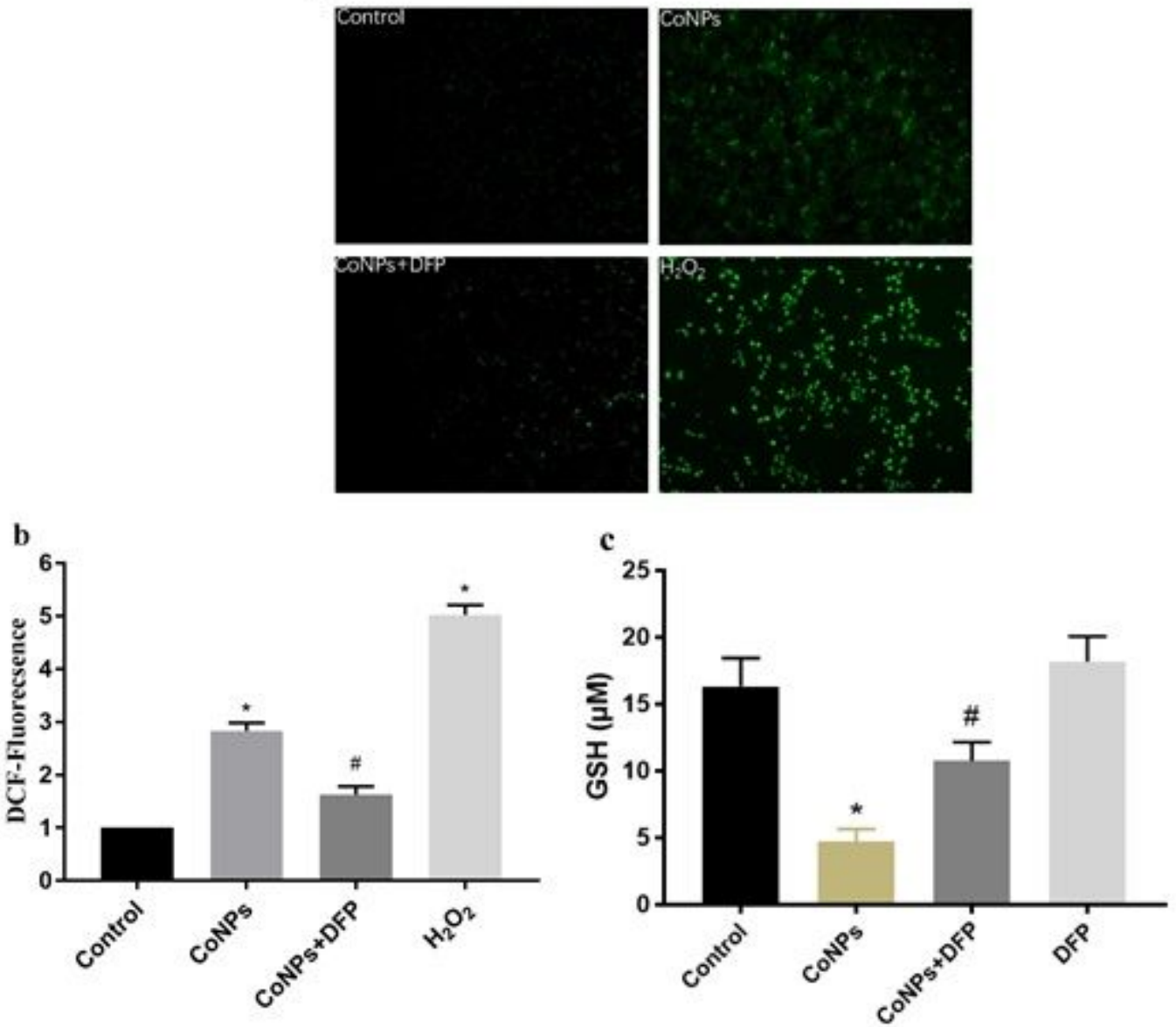

\section{Figure 3}

CoNPs treatment enhanced the level of intracellular ROS in MG-63 cells, while DFP significantly inhibited the formation of ROS. The cells were exposed to CoNPs $(400 \mu \mathrm{M})$ and DFP $(25 \mu \mathrm{M})$ for $24 \mathrm{~h}$. a Representative microphotographs (magnification, $x 40$ ) demonstrating CoNP-induced ROS generation in MG-63 cells treated with control, $400 \mu \mathrm{M}$ CoNPs, $400 \mu \mathrm{M}$ CoNPs $+25 \mu \mathrm{M}$ DFP and H2O2 (24 h). H2O2 group as positive control. $b$ The fluorescent intensity of cells with different treatments. $c$ Effects of CoNPs and DFP on GSH in MG-63 cells. *p $<0.05$ vs. control, \#p < 0.05 vs. CoNPs group. 

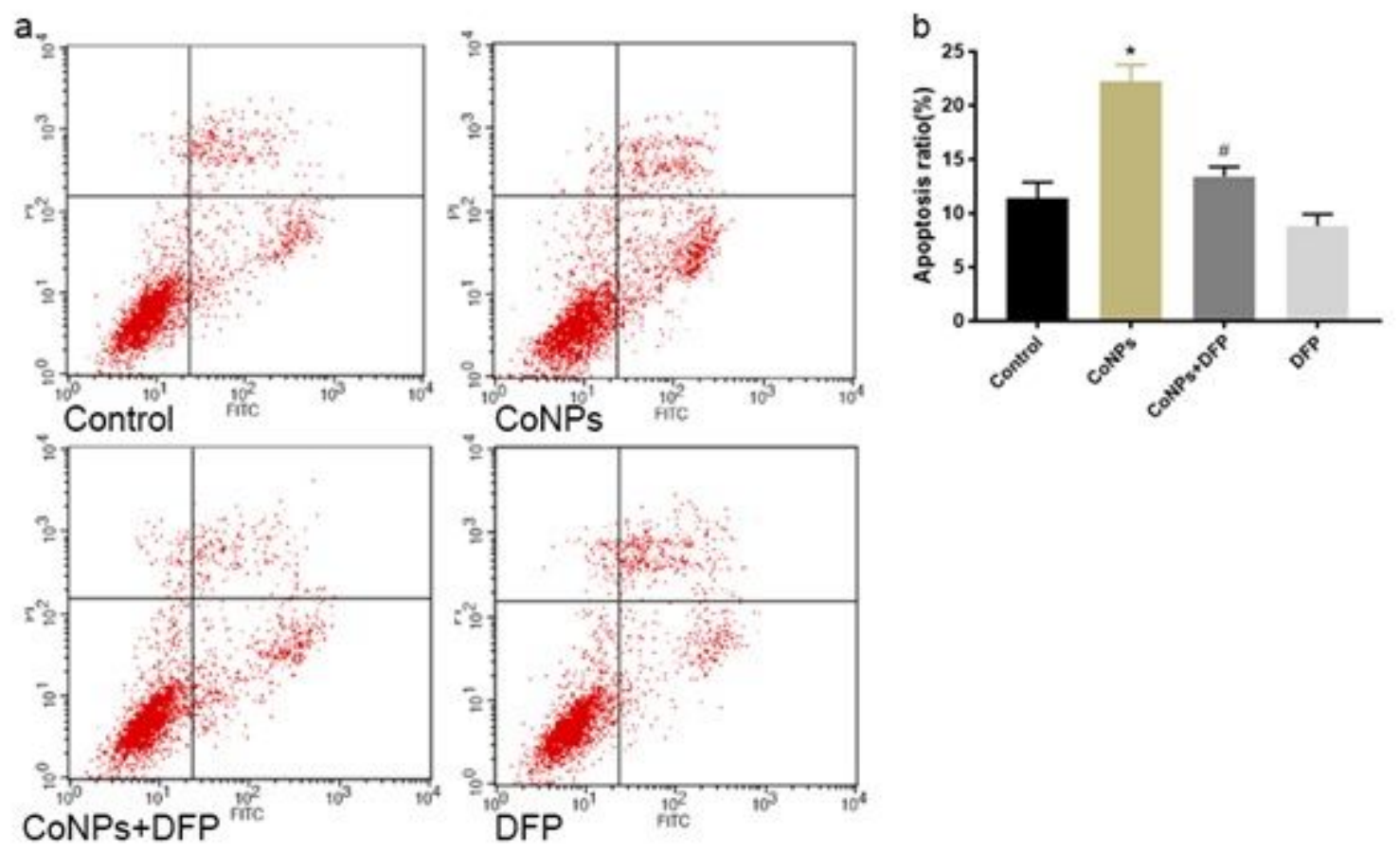

\section{Figure 4}

Cell apoptosis levels of MG-63 cells in presence of CoNPs and DFP. The cells were co-incubated with CoNPs $(400 \mu \mathrm{M})$ and DFP $(25 \mu \mathrm{M})$ for $24 \mathrm{~h}$. a The apoptosis was detected by flow cytometry. b Quantification of MG-63 cells apoptosis. All the data were expressed as mean \pm SD of three independent experiments performed in triplicates. ${ }^{\star} p<0.05$ vs. control, $\# p<0.05$ vs. CoNPs group.
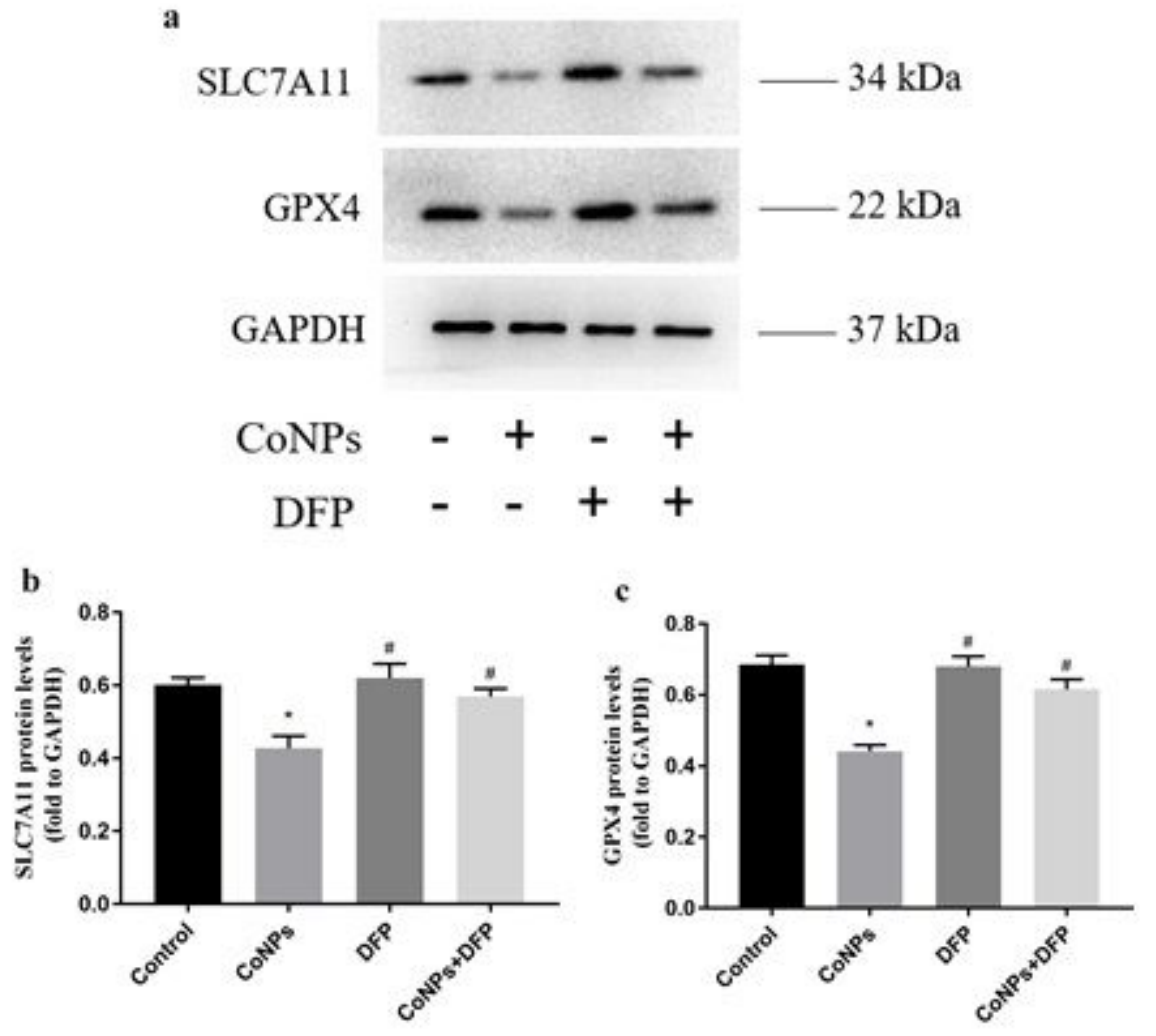
Figure 5

Effects of DFP on the protein levels of GPX4 and SLC7A11 in MG-63 cells after exposed to CoNPs. a Expressions of GPX4 and SLC7A11 were evaluated by western blotting. b Quantification of GPX4 and SLC7A11 expressions. ${ }^{*} p<0.05$ vs. control, $\# p<0.05$ vs. CoNPs group. 